\title{
Transcatheter closure of a ruptured aneurysm of the sinus of Valsalva
}

\author{
Seamus Cullen, Jane Somerville, Andrew Redington
}

\begin{abstract}
A ruptured aneurysm of the sinus of Valsalva in a man of 34 was closed by a transcatheter technique with a $12 \mathrm{~mm}$ modified Rashkind umbrella device.
\end{abstract}

(Br Heart f 1994;71:479-480)

Rupture of an aneurysm of the sinus of Valsalva is rare and is more likely to occur in adolescence or adult life. ${ }^{1}$ It is usually caused by a congenital weakness of the wall of the sinus which leads to progressive dilatation and ultimately rupture into one of the four chambers of the heart or into the mediastinum.

Cross sectional echocardiography can accurately demonstrate the defect, which can be corrected surgically without recourse to cardiac catheterisation. ${ }^{2}$ After a successful correction the long-term prognosis is usually good, though aortic regurgitation and recurrence of lesions have been reported.

We describe successful transcatheter closure of a ruptured sinus of Valsalva.

\section{Case report}

A 34 year old man was referred to this hospital with a three month history of increasing breathlessness on exertion and of fatigue. Ten years before he had had surgery elsewhere for a ruptured aneurysm of the sinus of Valsalva. He had been symptom free with no murmurs until this presentation. He had a grade $4 / 6$ continuous murmur best heard at the midsternal edge. A standard 12 lead electrocardiogram showed sinus rhythm, left atrial enlargement, and left ventricular hypertrophy. Cross sectional echocardiography showed the usual atrial arrangement (situs solitus), concordant connections, and a left-sided aortic arch. The aortic root was dilated $(4 \cdot 7 \mathrm{~cm})$. Colour Doppler examination showed a high velocity jet originating from the right coronary sinus and entering the right ventricle. There was trivial aortic incompetence. Cardiac catheterisation was performed under general anaesthesia. The pulmonary artery pressure was normal but there was a pulmonary to systemic shunt (2:1) with a step up in oxygen saturation in the right ventricular outflow tract. An aortogram showed contrast passing freely from the right coronary sinus into the right ventricle and filling the right ventricular outflow tract (figure).

\section{PROCEDURE}

Both femoral arteries were entered percutaneously with 7 French introducers. The right femoral vein was entered with an 8 French introducer. Heparin (100 units/kg) was given during the procedure and one dose of flucloxacillin and gentamicin was given. The fistula was shown by aortography and the right ventricle was entered retrogradely from the aorta with a 7 French multi-purpose end hole catheter. This was exchanged for an 0.035 inch guidewire over which an 8 French Mullins transseptal sheath was advanced into the right ventricle. A $12 \mathrm{~mm}$ modified umbrella device ${ }^{3}$ was passed through the sheath and deployed with ease, avoiding the coronary ostia. Positioning of the device was assisted by repeated injections of contrast medium into the aortic root via the catheter advanced from the contralateral leg. The final aortogram showed a trivial leak across the device and no aortic regurgitation (figure). The procedure took 57 minutes and no murmurs were audible after the device was in place.

Repeat cross sectional echocardiography the next day showed trivial aortic regurgitation and no leak across the device. The patient was discharged from hospital. He was symptom free six months later. We recommended a short period of anticoagulation and advised life-long antibiotic prophylaxis.

\section{Discussion}

Although the Rashkind umbrella was designed to close the arterial duct, ${ }^{4}$ we and others have used it to close atrial and ventricular septal defects and major aorto-pulmonary collateral arteries. ${ }^{35}$ Hourihan et al used the device to prevent valvar and paravalvar leaks. ${ }^{6}$

Rupture of the sinus of Valsalva is a rare lesion and in patients with symptoms it must be surgically closed during cardiopulmonary bypass. The long-term results of successful operation are usually good but aortic regurgitation can develop or the lesion can recur. We presume that a recurrence explains the new presentation in our patient. Repeat sternotomy was clearly undesirable and in view of the apparently ideal position of the defect, we decided to attempt closure with a transcatheter umbrella. Transcatheter closure of this lesion has not been described before, although Lock's group reported successful anterograde transcatheter closure of an aortato-right-ventricular fistula after aortic valve 

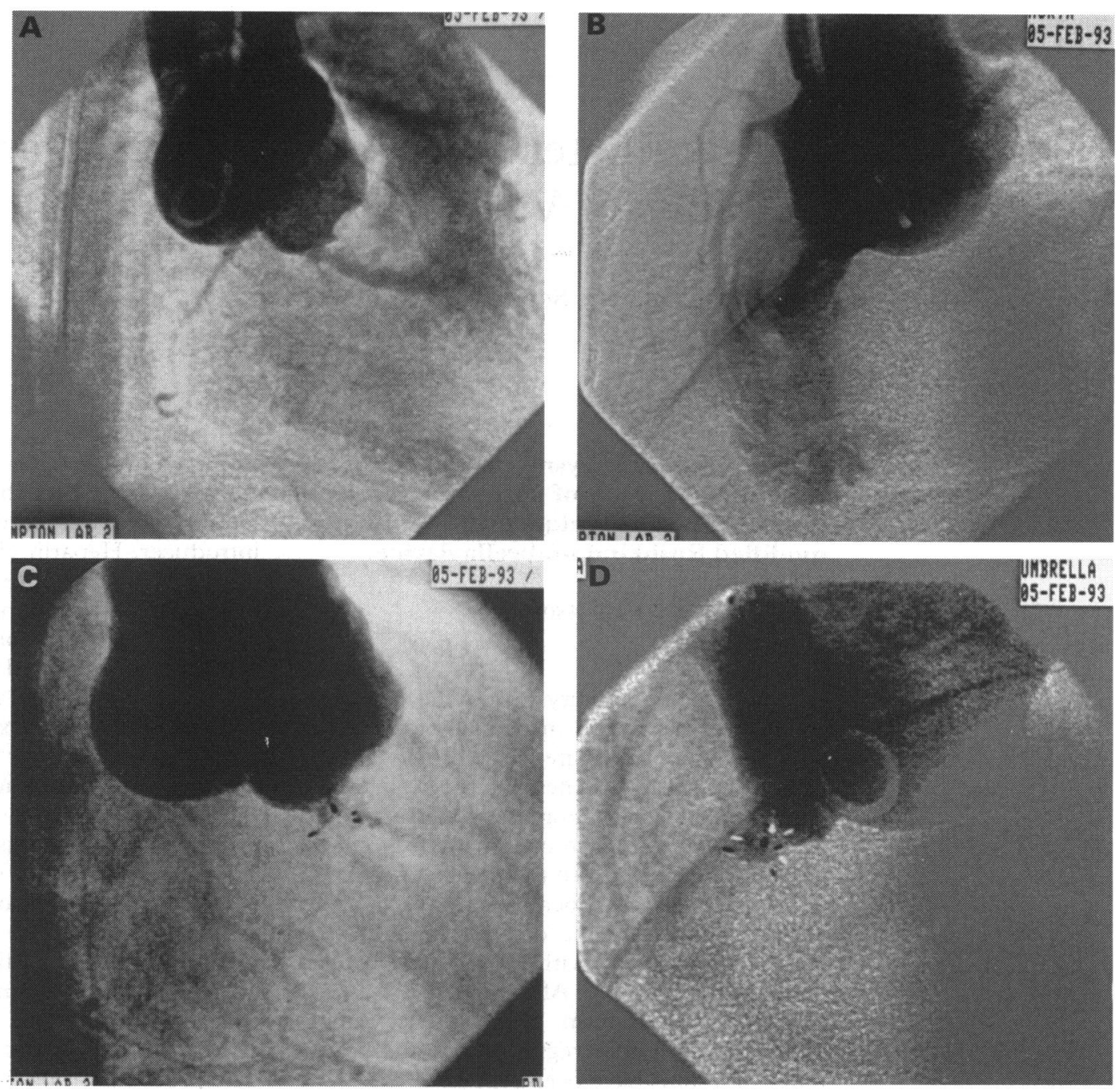

Left anterior oblique $(A)$ and lateral $(B)$ views of aortogram showing a fistula between right coronary sinus and right ventricle. Left anterior oblique (C) and lateral (D) views of repeat aortogram with umbrella device in place showing no residual defect.

replacement. ${ }^{6}$ In their patient closure was complicated and was successful after three attempts. The patient was clinically well 32 months after the final intervention. He was in NYHA congestive heart failure class 1 . They preferred an anterograde venous approach and snared a wire passed retrogradely through the defect. We think that this may unnecessarily complicate the procedure. A retrograde arterial approach, as used in our patient, facilitates accurate placement of the umbrella device and should significantly shorten the procedure time. This approach should at least be considered in future cases.

We and others have emphasised the benefits of transoesophageal echocardiography for transcatheter closure of intracardiac lesions. However, in our patient single plane transoesophageal echocardiography did not help us to position the device. Longitudinal scanning may have been more useful but was not available at the time of study. We decided to place a second catheter in the aortic root via the contralateral femoral artery, through which we repeatedly injected small amounts of contrast material to outline the defect during deployment of the device. The long-term risk of infective endocarditis is unknown, it seems prudent, however, to advise life-long antibiotic prophylaxis in patients with devices in this position. Because the device may be a source of systemic emboli we advise shortterm anticoagulation during the period of endothelialisation.

This instance of successful transcatheter closure of a ruptured sinus of Valsalva aneurysm extends the indications for use of the umbrella device.

1 Goldberg N, Krasnon N. Sinus of Valsalva aneurysms. Clin Cardiol 1990;13:831-6.

2 Sahasakul Y, Panchavinnin P, Chaitharaphan S, Sakiyalak P. Echocardiographic diagnosis of a ruptured sinus of Valsalva:operation without catheterisation in seven patients. Br Heart $\mathcal{F}$ 1990;64: 195-8.

3 Redington AN, Rigby ML. Novel uses of the Rashkind ductal umbrella in adults and children with congenital heart disease. Br Heart f 1993;69:47-51.

4 Rashkind WJ, Cuaso CC. Transcatheter closure of a patent ductus arteriosus:successful use in a $3.5 \mathrm{~kg}$ infant. Pediatr Cardiol 1979;1:3-7

5 Hellenbrand WE, Mullins CE. Catheter closure of congenital cardiac defects. Cardiol Clin 1989;7:351-68.

6 Hourihan M, Perry SB, Mandell VS, Keane JF, Rome JJ, Bittl JA, Lock JE. Transcatheter umbrella closure of Bittl JA, Lock JE. Transcatheter umbrella closure of
valvular and paravalvular leaks. $f$ Am Coll Cardiol 1992; 6:131-7. 